\title{
Correction to: Effect of endometrial mechanical stimulation in an unselected population undergoing in vitro fertilization: futility analysis of a double-blind randomized controlled trial
}

\author{
Ashley M. Eskew ${ }^{1} \cdot$ Lauren D. Reschke ${ }^{1} \cdot$ Candice Woolfolk $^{1} \cdot$ Maureen B. Schulte ${ }^{2}$ Christina E. Boots ${ }^{3}$. \\ Darcy E. Broughton ${ }^{1} \cdot$ Patricia T. Jimenez ${ }^{1} \cdot$ Kenan R. Omurtag $^{1} \cdot$ Sarah L. Keller $^{1} \cdot$ Valerie S. Ratts $^{1} \cdot$ Randall R. Odem $^{1}$. \\ Emily S. Jungheim ${ }^{1}$
}

Published online: 17 May 2019

(C) Springer Science+Business Media, LLC, part of Springer Nature 2019

\section{Correction to: Journal of Assisted Reproduction and Genetics (2019) 36:299-305} https://doi.org/10.1007/s10815-018-1356-5

The original version of this article unfortunately contained mistakes. The complete list of corrections is given below.

\section{Page 1: Abstract, Results section}

One hundred women enrolled and were randomized from 2013 to 2017. Enrollment was terminated after futility analysis showed no difference in clinical pregnancy between EMS versus control, 47.2 versus $59.6 \%$ (OR 0.61, $95 \%$ CI $0.27-1.34, p=0.22$ ). There were no significant differences between women who underwent EMS and those who did not in terms of positive pregnancy test

The online version of the original article can be found at https://doi.org/ 10.1007/s10815-018-1356-5

Ashley M. Eskew

ashley.m.eskew@gmail.com

1 Department of Obstetrics and Gynecology, Washington University School of Medicine, 4444 Forest Park Avenue, St. Louis, MO 63108, USA

2 Reproductive Medical Associates of St. Louis, 641 N New Ballas, Creve Coeur, MO 63141, USA

3 Department of Obstetrics and Gynecology, Division of Reproductive Endocrinology and Infertility, Northwestern University, 259 E. Erie Street, Chicago, IL 60611, USA
54.7 versus $61.7 \%$ (OR $0.75,95 \%$ CI $0.34-1.67$, $p=0.48$ ), miscarriage 7.5 versus $2.1 \%$ (OR $3.7695 \%$ CI $0.41-34.85, p=0.22$ ), or live birth 43.4 versus $59.6 \%$ (OR $0.5295 \%$ CI $0.24-1.15, p=0.12$ ).

\section{Page 4: Results section, second paragraph}

Using an intention-to-treat approach, there were no statistically significant differences between the two groups when comparing EMS versus the control group in terms of positive pregnancy test 54.7 versus $61.7 \%$ (odds ratio (OR) 0.75 , 95\% confidence interval $(\mathrm{CI}) 0.34-1.67, p=0.48)$, clinical pregnancy 47.2 versus $59.6 \%$ (OR $0.61,95 \%$ CI $0.27-1.34$, $p=0.22$ ), miscarriage 7.5 versus $2.1 \%$ (OR $3.7695 \% \mathrm{CI}$ $0.41-34.85, p=0.22$ ), or live birth 43.4 versus $59.6 \%$ (OR $0.5295 \%$ CI $0.24-1.15, p=0.12$ ) (Table 3). Implantation rate was calculated using chi-square for equality of proportions and was noted to be lower in the EMS group, but not statistically significantly different than the control group, 33 versus $47 \%(p=0.09)$. Analysis with a per-protocol approach including only patients that underwent an embryo transfer yielded similar results when comparing EMS versus sham biopsy in terms of clinical pregnancy, miscarriage, and live birth rate (Table 4).

\section{Page 4: Table 2, fifth column, second row}

$0.34-1.67$

Publisher's note Springer Nature remains neutral with regard to jurisdictional claims in published maps and institutional affiliations. 